\section{The isomorphism of Minkowski space and the separable complex Hilbert space and its physical interpretation}

\author{
Vasil Penchev, vasildinev@gmail.com \\ Bulgarian Academy of Sciences: \\ Institute of Philosophy and Sociology \\ Dept. of Logical Systems and Models
}

Abstract. An isomorphism is built between the separable complex Hilbert space (quantum mechanics) and Minkowski space (special relativity) by meditation of quantum information (i.e. qubit by qubit). That isomorphism can be interpreted physically as the invariance between a reference frame within a system and its unambiguous counterpart out of the system. The same idea can be applied to Poincaré's conjecture (proved by G. Perelman) hinting another way for proving it, more concise and meaningful physically. Mathematically, the isomorphism means the invariance to choice, the axiom of choice, well-ordering, and well-ordering "theorem" (or "principle") and can be defined generally as "information invariance".

Key words: axiom of choice, choice, Hilbert space, Minkowski space, Poincaré's conjecture, quantum information, qubit, well-ordering

The set of all complex numbers, $\boldsymbol{C}$ is granted. Then the corresponding set of all subsets of $\boldsymbol{C}$ is the separable complex Hilbert space $\mathcal{H}$.

There is one common and often met identification of $\mathcal{H}$ with the set $\mathbb{H}$ of all ordinals of $\mathcal{H}$, which rests on the identification of any set with its ordinal. However, if any ordinal is identified as a certain natural number, and all natural numbers in Peano arithmetic are finite ${ }^{1}, \mathcal{H}$ and $\mathbb{H}$ should not be equated, for $\mathcal{H}$ includes actually infinite subsets ${ }^{2}$ of $2^{C}$. Here "actually infinite subset" means "set infinite in the sense of set theory".

Furthermore, $\mathcal{H}$ is identified as the set $\mathbf{H}$ of all well-ordered sets, the elements of which are elements of some set of $2^{C}$, i.e. in other words, the elements of $2^{C}$ considered as classes of equivalency by ordering are differed by ordering within any class of that ordering.

Those distinctions can be illustrated by the two basic interpretations of $\mathcal{H}:(1)$ as the vectors of $\mathrm{n}$-dimensional complex generalization of the usual 3D real Euclidean space, isomorphic to $\mathbf{H}$, and (2) as the squarely integrable functions, isomorphic to $\mathcal{H}$. The latter adds to the former unitarity (unitary invariance), which is usually interpreted as energy conservation

\footnotetext{
${ }^{1}$ This is a property implied by the axiom of induction.

${ }^{2}$ Here "actually infinite subset" means "set infinite in the sense of set theory".

31 is finite. The successor of any finite natural number is finite Consequently, all natural numbers are finite for the axiom of induction.
}

in their application in quantum mechanics. Back seen, energy conservation is a physical equivalent of both (3) equivalence after ordering and (4) actual infinity, i.e. to (5) the concept of ordinal number in set theory.

On the contrary, once one does not involves energy conservation, e.g. generalizing it to energy-momentum conservation as in the theory of general relativity or that of entanglement, $\mathbf{H}$ rather than $\mathcal{H}$ is what should be used unlike quantum mechanics based on $\mathcal{H}$, and actual infinity avoided or at least precisely thought before utilizing.

Furthermore, (6) the relation between $\mathcal{H}$ and $\boldsymbol{H}$ can be interpreted as the 3D Euclidean space under (7) the additional condition of cyclicality (reversibility) of $\boldsymbol{H}$ conventionally identifying the first "infinite" element with the "first" element of any (trans)finite well-ordering. Indeed, the axiom of induction in Peano arithmetic does not admit infinite natural numbers ${ }^{3}$. If one needs to reconcile both finite and transfinite induction to each other, the above condition is sufficient.

It should be chosen for Poincaré's conjecture [34] proved by G. Perelman [35-37]. If that condition misses, the topological structure is equivalent to any of both almost disjunctive domains $^{4}$ of Minkowski's space of special relativity ${ }^{5}$ rather than to a 4D Euclidean ball. The two domains of Minkowski space $\mathcal{M}$ can be interpreted as two opposite, "causal directions" resulting in both reversibility of the 3D Euclidean space and topological structure of the above 4D ball.

The relation between $\mathcal{H}$ and $\boldsymbol{H}$ generates any of the two areas of $\mathcal{M}$ as follows. Both unitarity of $\mathcal{H}$ and non-unitarity of $\boldsymbol{H}$ for any ordinal $n$ and any well-ordering of length $n$ are isomorphic to a 3D Euclidean sphere ${ }^{6}$ with the radius $r(n)$. All those spheres represent the area at issue.

That construction can be interpreted physically as well. Energy (E) conservation as unitarity represents the class of equivalence of any ordinal $n$. If the concept of physical force (F) is introduced as any reordering, i.e. the relation between any two elements of the above class, it can be reconciled with energy conservation (unitarity) by the quantity of distance (x) in units of elementary permutations for the reordering so that $F \cdot x=E$.

Back seen, both (6) and (7) implies Poincaré's conjecture and thus offer another way of its proof.

One can discuss the case where $\mathcal{H}$ is identified with $\boldsymbol{H}$ and what it implies. Then (8) the axiom of induction in Peano arithmetic should be replaced by transfinite induction correspondingly to (4) above, and (9) the statistical ensemble of well-orderings (as after measurement in quantum mechanics) should be equated to the set of the same elements (as the coherent state before measurement in quantum mechanics) for (3) above.

In fact, that is the real case in quantum mechanics for unitarity as energy conservation is presupposed. Then (8) implies the theorems of absence of hidden variables in quantum mechanics [1], [2], i.e. a kind of mathematical completeness interpretable as the completeness of quantum mechanics vs.

\footnotetext{
${ }^{4}$ They are almost disjunctive as share the light cone.

5 Indeed, special relativity is a causal theory, which excludes the reverse causality implied by cyclicality.

6 This means the surface of a 3D Euclidean ball.
} 
Einstein, Podolsky, and Rosen's hypothesis of the incompleteness of quantum mechanics [3]:

The (8) and (9) together imply the axiom of choice. Indeed, the coherent state (the unordered set of elements) excludes any well-ordering for the impossibility of hidden variables implied by (8). However, it can be anyway well-ordered for (9). This forces the well-ordering principle ("theorem") to be involved, which in turn is equivalentto the axiom of choice.

Furthermore, $\mathcal{H}$ can be represented as all sets of qubits.

A qubit is defined in quantum mechanics and information as the (10) normed superposition of two orthogonal ${ }^{7}$ subspaces of $\mathcal{H}$ :

$$
Q \stackrel{\text { def }}{=} \alpha|0\rangle+\beta|1\rangle
$$

$|0\rangle,|1\rangle$ are the two orthogonal subspaces of $\mathcal{H}$.

$\alpha, \beta \in C:|\alpha|^{2}+|\beta|^{2}=1$.

Then, (11) Q is isomorphic to a unit 3D Euclidean ball, in which two points in two orthogonal great circles ate chosen so that the one of them (the corresponding to the coefficient $\beta$ ) is on the surface of the ball.

That interpretation is obvious mathematically. It makes sense physically and philosophically for the above consideration of space as the relation of $\mathcal{H}$ and $\boldsymbol{H}$.

Now, it can be slightly reformulated and reinterpreted as the joint representability of $\mathcal{H}$ and $\boldsymbol{H}$, and thus their unifiability in terms of quantum information.

Particularly, any theory of quantum information, including quantum mechanics as far as it is so representable, admits the coincidence of model and reality: right a fact implied by the impossibility of hidden variables in quantum mechanics for any hidden variable would mean a mismatch of model and reality.

$\boldsymbol{H}$ can be interpreted as an equivalent series of qubits for any two successive axes of $\boldsymbol{H}$ are two orthogonal subspaces of $\mathcal{H}$ :

$\left\{C_{j}\right\} \in \boldsymbol{H}$; then (12) any successive pair $\left(C_{j}, C_{j+1}\right)=Q_{j+1}$; $Q_{j+1} \in \boldsymbol{Q}$ under the following conditions:

$$
\text { (13) } \alpha_{j+1}=\frac{C_{j}}{\sqrt{\left(C_{j}\right)^{2}+\left(C_{j+1}\right)^{2}}} ; \beta_{j+1}=\frac{C_{j+1}}{\sqrt{\left(C_{j}\right)^{2}+\left(C_{j+1}\right)^{2}}} ;
$$

(14) $\alpha_{1}=0 ; \beta_{1}=\frac{C_{1}}{\left|C_{1}\right|}$;

(15) If both $C_{j}, C_{j+1}=0, \alpha_{j+1}=0, \beta_{j+1}=1$.

(14) and (15) are conventional, chosen rather arbitrarily only to be conserved a one-to-one mapping between $\boldsymbol{H}$ and $\boldsymbol{Q}$.

$\boldsymbol{Q}$ is intendedly constructed to be ambivalent to unitarity for any qubit is internally unitary, but the series of those is not. Furthermore, one can define n-bit where a qubit is 2-bit therefore transforming unitarily any non-unitary n-series of complex numbers. The essence of that construction is the double conservation between the two pairs: "within - out of" and "unitarity - nonunitarity".

That conservation is physical and informational, in fact. The simultaneous choice between many alternatives being unitary and thus physically interpretable is equated to a series of elementary or (at least more elementary) choices. Then, the visible as physical inside will look like the "chemical" outside and vice versa. If a wholeness such as the universe is defined to contain internally its externality, this can be modeled anyway consistently equating the non-unitary "chemical" and unitary "physical" representations in the framework of a relevant physical and informational conservation.

$\mathcal{H}$ can be furthermore interpreted as all possible pairs of characteristic functions of independent probability distributions and thus, of all changes of probability distributions of the state of a system, e.g. a quantum system.

Practically all probability distributions and their characteristic functions of the states of real systems are continuous and even smooth as usual. The neighboring values of probability implies the neighborhood of the states. Thus the smoothness of probability distribution implies a well-ordering and by the meditation of it, a kind of causality: the probability of the current state cannot be changed jump-like.

This is an expression of a deep mathematical dependence (or invariance) of the continuous (smooth) and discrete. The probability distribution can mediate between them as follows:

$\mathcal{H}$ can be defined as the sets of the ordinals of $\boldsymbol{H}$ where a representative among any subset of the permutations (wellorderings) of $n$ elements is chosen according a certain and constructive rule. That rule in the case in question is to be chosen that permutation (well-ordering), the probability distribution of which is smooth.

Particularly, the homotopy of $\boldsymbol{H}$ can identified with, and thus defined as that mapping of $\boldsymbol{H}$ into $\mathcal{H}$ conserving the number of elements, i.e. the dimensionality $n$ of the vector between $\boldsymbol{H}$ and $\mathcal{H}$. If $\boldsymbol{H}$ is interpreted as the set of types on $\boldsymbol{C}$, this implies both "axiom of univalence" [4] and an (iso)morphism between the category of all categories and the pair of $\mathcal{H}$ and $\boldsymbol{H}$.

That consideration makes obvious the equivalence of the continuous (smooth) and discrete as one and the same wellordering chosen as an ordinal among all well-orderings (permutations) of the same elements and it by itself accordingly. In other words, the continuous (smooth) seems to be class of equivalence of the elements of a set (including finite as a generalization of continuity as to finite sets).

Furthermore, the same consideration can ground (3) and (9) above, i.e. the way, in which a coherent state before measurement is equivalent of the statistical ensemble of measured states in quantum mechanics. The same property can be called "invariance to choice" including the invariance to the axiom of choice particularly.

This means that the pure possibility, e.g. that of pure existence in mathematics, also interpretable as subjective probability should be equated to the objective probability of the corresponding statistical ensemble once unitarity (energy conservation) has already equated $\mathcal{H}$ and $\boldsymbol{H}$.

Indeed, the set or its ordinal can be attributed to the elements of $\mathcal{H}$ and the statistical mix of all elements of $\boldsymbol{H}$ corresponding to a given element of $\mathcal{H}$. Any measurement ascribes randomly a certain element of the corresponding subset of $\boldsymbol{H}$ to any given element of $\mathcal{H}$. Thus measurement is not unitary, e.g. a ,collapse of wave function".

Then, $\mathcal{H}$ and $\boldsymbol{H}$ can be interpreted as two identical but complementary dual spaces of the separable complex Hilbert space. Initarity means right their identity, and the non-unitarity of measurement representing a random choice means their complementarity.

\footnotetext{
${ }^{7}$ Any two disjunctive subspaces of $\mathcal{H}$ are orthogonal to each other.
} 
That "invariance to choice" can ground both so-called Born probabilistic [5] and Everett (\& Wheeler) "many-worlds" interpretations of quantum mechanics [6], [7], [8]. The former means the probability for a state to be measured or a "world" to take place, and the former complement that consideration by the fact that all elements constituting the statistical ensemble can be consistently accepted as actually existing.

One can emphasize that the Born interpretation ascribes a physical meaning of the one component (namely the square of the module as probability) of any element of the field of complex numbers underlying both $\mathcal{H}$ and $\boldsymbol{H}$. After that, the physical meaning of the other component, the phase is even much more interesting. It should correspond to initarity, and then, it seems to be redundant, i.e. the field of real numbers would be sufficient, on the one hand, but furthermore, to time, well-ordering, and choice implied by it.

In other words, just the phase is what is both physical and mathematical "carrier" and "atom" of the invariance of choice featuring the separable complex Hilbert space.

\section{REFERENCES}

[1] Neuman, J. von. Mathematische Grundlagen der Quantenmechanik. Berlin: Springer, pp. 167-173 (1932).

[2] Kochen, S., Specker, E. "The Problem of Hidden Variables in Quantum Mechanics," Journal of Mathematics and Mechanics 17(1): 59-87 (1968).

[3] Einstein, A., Podolsky, B., Rosen, N. "Can Quantum-Mechanical Description of Physical Reality Be Considered Complete?" Physical Review 47(10): 777-780.

[4] (Institute for Advanced Study, Princeton, NJ, Univalent Foundations Program) Homotopy type theory: univalent foundations of mathematics. Princeton, NJ: Lulu Press, Univalent Foundations Program (2013).

[5] Born, M. "Max Born - Nobel Lecture: The Statistical Interpretations of Quantum Mechanics," Nobelprize.org. Nobel Media AB 2014. Web. (13 Jul 2016.)

$<$ http://www.nobelprize.org/nobel_prizes/physics/laureates/1954/born -lecture.html>

[6] Everett III, H. "Relative State" Formulation of Quantum Mechanics," Reviews of Modern Physics 29(3): 454-462 (1957).

[7] DeWitt, B. S., Graham, N. (eds.) The many-worlds interpretation of quantum mechanics: a fundamental exposition. Princeton, NJ: University Press (1973)

[8] Wheeler, J. A., Zurek, W. H. (eds.). Quantum theory and measurement. Princeton, N.J.: Princeton University Press, 1983. 